\title{
Convergence Theorems of Common Elements for Pseudocontractive Mappings and Monotone Mappings
}

\author{
Jae Ug Jeong \\ Department of Mathematics, Dongeui University, Busan 614-714, Republic of Korea \\ Correspondence should be addressed to Jae Ug Jeong; jujeong@deu.ac.kr
}

Received 28 July 2014; Accepted 8 September 2014

Academic Editor: Xiaolong Qin

Copyright (C) 2015 Jae Ug Jeong. This is an open access article distributed under the Creative Commons Attribution License, which permits unrestricted use, distribution, and reproduction in any medium, provided the original work is properly cited.

An algorithm for treating pseudocontractive mappings and monotone mappings is proposed. Convergence analysis of algorithm is investigated in the framework of Hilbert spaces.

\section{Introduction}

The motivation for common element problem is mainly due to its possible applications to mathematical modeling of concrete complex problems. The common element problems include mini-max problems, complementarily problems, equilibrium problems, common fixed point problems, and variational inequalities as special cases; see [1-7] and the references therein. It is well-known that the convex feasibility problem is a special case of the common zero (fixed) points of nonlinear mappings. And many important problems have reformulations which require finding zero points, for instance, evolution equations, complementarily problems, mini-max problems, and variational inequalities and optimization. For studying zero points of monotone mappings, the most well-known algorithm is the proximal point algorithm; see $[8,9]$ and the references therein. Regularization methods recently have been investigated for treating zero points of monotone mappings; see $[2,5,6,9]$ and references therein.

In 2010, Takahashi et al. [6] studied zero point problems of the sum of two monotone mappings and fixed point problems of a nonexpansive mapping based on the following iterative algorithm:

$$
\begin{gathered}
x_{1}=x \in C, \\
y_{n}=\alpha_{n} x+\left(1-\alpha_{n}\right) J_{r_{n}}\left(I-r_{n} A\right) x_{n}, \\
x_{n+1}=\beta_{n} x_{n}+\left(1-\beta_{n}\right) T y_{n}, \quad \forall n \geq 1,
\end{gathered}
$$

where $C$ is a nonempty closed convex subset of a real Hilbert space $H,\left\{\alpha_{n}\right\}$ and $\left\{\beta_{n}\right\}$ are real number sequences in $(0,1)$, $\left\{r_{n}\right\}$ is a positive sequence, $T: C \rightarrow C$ is a nonexpansive mapping, $A: C \rightarrow H$ is an inverse strongly monotone mapping, $B: H \rightarrow 2^{H}$ is a maximal monotone mapping, and $J_{r_{n}}=\left(I+r_{n} B\right)^{-1}$, where $I$ is the identity mapping. They proved that the sequence $\left\{x_{n}\right\}$ generated in (1) converges strongly to some $z \in F(T) \cap(A+B)^{-1}(0)$ provided that the control sequences satisfy some restrictions, where $F(T)$ is the set fixed points of $T$.

In 2014, Shahzad and Zegeye [5] considered an iterative method for a common point of fixed points of Lipschitzian pseudocontractive mappings and zeros of sum of two monotone mappings based on the projection method in a real Hilbert space. To be more precise, they investigated the following algorithm:

$$
\begin{gathered}
x_{0} \in C, \\
y_{n}=\left(1-\beta_{n}\right) x_{n}+\beta_{n} T x_{n},
\end{gathered}
$$$$
x_{n+1}
$$$$
=P_{C}\left[\left(1-\alpha_{n}\right)\left(\theta_{n} x_{n}+\delta_{n} T y_{n}+\gamma_{n} J_{r_{n}}\left(I-\lambda_{n} A\right) x_{n}\right)\right] \text {, }
$$

where $C$ is a nonempty closed convex subset of a real Hilbert space $H,\left\{\alpha_{n}\right\},\left\{\beta_{n}\right\},\left\{\theta_{n}\right\},\left\{\delta_{n}\right\}$, and $\left\{\gamma_{n}\right\}$ are real number sequences in $(0,1),\left\{r_{n}\right\}$ is a positive sequence, $T: C \rightarrow C$ is a Lipschitzian pseudocontractive mapping, $A: C \rightarrow H$ is an inverse strongly monotone mapping, $B: H \rightarrow 2^{H}$ 
is a maximal monotone mapping, and $J_{r_{n}}=\left(I+r_{n} B\right)^{-1}$. They proved that the sequence $\left\{x_{n}\right\}$ generated in (2) converges strongly to the minimum-norm point $x \in F(T) \cap(A+B)^{-1}(0)$ provided that the control sequences satisfy some restrictions.

In this paper, we are concerned with the problem of finding a common element in the intersection $F\left(T_{1}\right) \cap F\left(T_{2}\right) \cap$ $(A+B)^{-1}(0)$, where $F\left(T_{i}\right)$ denotes the fixed point set of the pseudocontractive mapping $T_{i}, i=1,2$, and $(A+$ $B)^{-1}(0)$ denotes the zero point set of the sum of an inverse strongly monotone mapping $A$ and a maximal monotone mapping $B$. Applications to a common element of the set of common fixed points of Lipschitzian pseudocontractive mappings and solutions of variational inequality for $\alpha$-inverse strongly monotone mappings are included. Our theorems improve and extend those announced by Shahzad and Zegeye [5], Takahashi et al. [6], and other authors with the related interest.

\section{Preliminaries}

Let $H$ be a real Hilbert space with the inner product $\langle\cdot, \cdot\rangle$ and the norm $\|\cdot\|$. Let $C$ be a nonempty closed convex subset of $H$ and let $P_{C}$ be the metric projection from $H$ onto $C$. Let $T: C \rightarrow C$ be a mapping. In this paper, we use $F(T)$ to denote the fixed point set of $T$; that is, $F(T)=\{x \in C: x=T x\}$.

Recall that $T$ is nonexpansive if

$$
\|T x-T y\| \leq\|x-y\|, \quad \forall x, y \in C .
$$

$T$ is said to be a $\gamma$-strictly pseudocontractive mapping if there exists $\gamma \in[0,1)$ such that

$$
\begin{array}{r}
\|T x-T y\|^{2} \leq\|x-y\|^{2}+\gamma\|(I-T) x-(I-T) y\|^{2}, \\
\forall x, y \in C .
\end{array}
$$

Note that the class of $\gamma$-strictly pseudocontractive mappings includes the class of nonexpansive mappings as a special case. $T$ is said to be a pseudocontractive mapping if

$$
\begin{array}{r}
\|T x-T y\|^{2} \leq\|x-y\|^{2}+\|(I-T) x-(I-T) y\|^{2}, \\
\forall x, y \in C .
\end{array}
$$

We note that inequalities (4) and (5) can be equivalently written as

$$
\langle x-y, T x-T y\rangle \leq\|x-y\|^{2}-\gamma\|(I-T) x-(I-T) y\|^{2}
$$

for some $\gamma>0$ and

$$
\langle x-y, T x-T y\rangle \leq\|x-y\|^{2}, \quad \forall x, y \in C,
$$

respectively. Note that the class of $\gamma$-strictly pseudocontractive mappings is contained in the class of pseudocontractive mappings. We note that the inclusion is proper. We remark that $T$ is a $\gamma$-strictly pseudocontractive mapping if and only if $I-T$ is a $\gamma$-inverse strongly monotone mapping and $T$ is a pseudocontractive mapping if and only if $I-T$ is a monotone mapping.
Let $A: C \rightarrow H$ be a mapping and $A^{-1} 0$ stands for the zero point set of $A$; that is, $A^{-1} 0=\{x \in C: A x=0\}$. Recall that $A$ is said to be monotone if

$$
\langle A x-A y, x-y\rangle \geq 0, \quad \forall x, y \in C
$$

$A$ is said to be $\alpha$-inverse strongly monotone if there exists a constant $\alpha>0$ such that

$$
\langle A x-A y, x-y\rangle \geq \alpha\|A x-A y\|^{2}, \quad \forall x, y \in C .
$$

It is not hard to see that $\alpha$-inverse strongly monotone mappings are Lipschitz continuous with constant $L=1 / \alpha$; that is, $\|A x-A y\| \leq(1 / \alpha)\|x-y\|$ for all $x, y \in C$.

Recall that the classical variational inequality, denoted by $\operatorname{VI}(C, A)$, is to find $u \in C$ such that

$$
\langle A u, v-u\rangle \geq 0, \quad \forall v \in C .
$$

A multivalued mapping $B: H \rightarrow 2^{H}$ with the domain $D(B)=\{x \in H: B x \neq \phi\}$ and the range $R(B)=\{B x:$ $x \in D(B)\}$ is said to be monotone if, for $x_{1} \in D(B), x_{2} \in$ $D(B), y_{1} \in B x_{1}$, and $y_{2} \in B x_{2}$, we have $\left\langle x_{1}-x_{2}, y_{1}-y_{2}\right\rangle \geq$ 0 . A monotone mapping $B$ is said to be maximal if its graph $G(B)=\{(x, y): y \in B x\}$ is not properly contained in the graph of any other monotone mapping. Let $B: H \rightarrow 2^{H}$ be a maximal monotone mapping. Then we can define, for each $\lambda>0$, a nonexpansive single-valued mapping $J_{\lambda}: H \rightarrow H$ by $J_{\lambda}=(I+\lambda B)^{-1}$. It is called the resolvent of $B$. We know that $B^{-1} 0=F\left(J_{\lambda}\right)$ for all $\lambda>0$ and $J_{\lambda}$ is firmly nonexpansive.

Lemma 1. Let $H$ be a real Hilbert space. Then, for any given $x, y \in H$, the following inequality holds:

$$
\|x+y\|^{2} \leq\|x\|^{2}+2\langle y, x+y\rangle .
$$

Lemma 2 (see [10]). Let $C$ be a convex subset of a real Hilbert space $H$. Let $x \in H$. Then $x_{0}=P_{C} x$ if and only if

$$
\left\langle z-x_{0}, x-x_{0}\right\rangle \leq 0, \quad \forall z \in C \text {. }
$$

Lemma 3 (see [2]). Let $C$ be a nonempty closed convex subset of a real Hilbert space $H$. Let $A: C \rightarrow H$ be a mapping and let $B: H \rightarrow 2^{H}$ be a maximal monotone mapping. Then $F\left(J_{\lambda}(I-\right.$ $\lambda A))=(A+B)^{-1} 0$.

Lemma 4 (see [11]). Let $H$ be a Hilbert space. Let $B_{1}: D\left(B_{1}\right) \subseteq$ $H \rightarrow 2^{H}$ and let $B_{2}: D\left(B_{2}\right) \subseteq H \rightarrow 2^{H}$ be maximal monotone mappings. Suppose that $D(A) \cap \operatorname{int}(D(B)) \neq \phi$. Then $B_{1}+B_{2}$ is a maximal monotone mapping.

Lemma 5 (see [4]). Let $\left\{a_{n}\right\}$ be a sequence of real numbers. Assume that there exists a subsequence $\left\{n_{i}\right\}$ of $\{n\}$ such that $a_{n_{i}}<a_{n_{i}+1}$ for all $i \in \mathbb{N}$. Then there exists a nondecreasing sequence $\left\{m_{k}\right\} \subset \mathbb{N}$ such that $m_{k} \rightarrow \infty$ and the following properties are satisfied by all sufficiently large numbers $k \in \mathbb{N}$ :

$$
a_{m_{k}} \leq a_{m_{k}+1}, \quad a_{k} \leq a_{m_{k}+1}
$$


Lemma 6 (see [12]). Let $H$ be a real Hilbert space. Then, for all $x_{i} \in H$ and $\alpha_{i} \in[0,1]$ for $i=1,2, \ldots, n$ such that $\alpha_{1}+\alpha_{2}+$ $\cdots+\alpha_{n}=1$, the following equality holds:

$$
\begin{aligned}
\| \alpha_{0} x_{0} & +\alpha_{1} x_{1}+\cdots+\alpha_{n} x_{n} \|^{2} \\
& =\sum_{i=0}^{n} \alpha_{i}\left\|x_{i}\right\|^{2}-\sum_{0 \leq i, j \leq n} \alpha_{i}, \alpha_{j}\left\|x_{i}-x_{j}\right\|^{2} .
\end{aligned}
$$

Lemma 7 (see [7]). Let $C$ be a nonempty closed convex subset of a real Hilbert Hilbert and let $T: C \rightarrow C$ be a continuous pseudocontractive mapping. Then

(i) $F(T)$ is a closed convex subset of $C$;

(ii) $(I-T)$ is demiclosed at zero; that is, if $\left\{x_{n}\right\}$ is a sequence in $C$ such that $x_{n} \rightarrow x$ and $T x_{n}-x_{n} \rightarrow 0$ as $n \rightarrow \infty$, then $x=T x$.

Lemma 8 (see [13]). Let $\left\{a_{n}\right\}$ be a sequence of nonnegative real numbers satisfying the following relation:

$$
a_{n+1} \leq\left(1-\alpha_{n}\right) a_{n}+\alpha_{n} \delta_{n}, \quad n \geq n_{0},
$$

where $\left\{\alpha_{n}\right\} \subset(0,1)$ and $\left\{\delta_{n}\right\} \subset \mathbb{R}$ satisfy the following conditions: $\lim _{n \rightarrow \infty} \alpha_{n}=0, \sum_{n=1}^{\infty} \alpha_{n}=\infty$, and $\lim \sup _{n \rightarrow \infty} \delta_{n} \leq 0$. Then $\lim _{n \rightarrow \infty} a_{n}=0$.

\section{Main Results}

Theorem 9. Let $C$ be a nonempty closed convex subset of a real Hilbert space $H$. Let $T_{1}, T_{2}: C \rightarrow C$ be Lipschitzian pseudocontractive mappings with Lipschitz constants $L_{1}$ and $L_{2}$, respectively. Let $A: C \rightarrow H$ be an $\alpha$-inverse strongly monotone mapping and let $B$ be a maximal monotone mapping such that the domain of $B$ is subset of $C$. Assume that $\mathscr{F}=$ $F\left(T_{1}\right) \cap F\left(T_{2}\right) \cap(A+B)^{-1} 0 \neq \phi$. Let $J_{\lambda_{n}}=\left(I+\lambda_{n} B\right)^{-1}$, where $\left\{\lambda_{n}\right\}$ is a positive real number sequence. Given $x_{1}, u \in C$, let $\left\{x_{n}\right\}$ be the sequence generated by the following algorithm:

$$
\begin{gathered}
z_{n}=\left(1-c_{n}\right) x_{n}+c_{n} T_{2} x_{n}, \\
y_{n}=\left(1-\beta_{n}\right) x_{n}+\beta_{n} T_{1} x_{n}, \\
x_{n+1}=P_{C}\left[\alpha_{n} u+\left(1-\alpha_{n}\right)\right. \\
\times\left(\theta_{n} x_{n}+\delta_{n} T_{1} y_{n}+\gamma_{n} T_{2} z_{n}\right. \\
\left.\left.+\xi_{n} J_{\lambda_{n}}\left(I-\lambda_{n} A\right) x_{n}\right)\right] .
\end{gathered}
$$

Assume that the sequences $\left\{\alpha_{n}\right\},\left\{\beta_{n}\right\},\left\{c_{n}\right\},\left\{\theta_{n}\right\},\left\{\delta_{n}\right\},\left\{\gamma_{n}\right\},\left\{\xi_{n}\right\}$, and $\left\{\lambda_{n}\right\}$ satisfy the following restrictions:

(a) $0<a<\lambda_{n}<b<2 \alpha$;

(b) $0<c \leq \theta_{n}, \delta_{n}, \gamma_{n}, \xi_{n} \leq d<1$ and $\theta_{n}+\delta_{n}+\gamma_{n}+\xi_{n}=1$;

(c) $0<\alpha_{n}<e<1, \lim _{n \rightarrow \infty} \alpha_{n}=0$ and $\sum_{n=1}^{\infty} \alpha_{n}=\infty$;

(d) $\delta_{n}+\gamma_{n}+\xi_{n} \leq \beta_{n}, c_{n} \leq \beta<1 /\left(\sqrt{1+L^{2}}+1\right)$, for all $n \geq 1$, for some real numbers $a, b, c, d, e, \beta>0$, where $L=$ $\max \left\{L_{1}, L_{2}\right\}$. Then $\left\{x_{n}\right\}$ converges strongly to some point $\bar{x}$, where $\bar{x}=P_{\mathscr{F}} u$.

Proof. First, we show that $I-\lambda_{n} A$ is nonexpansive. Indeed, we have

$$
\begin{aligned}
\|(I- & \left.\lambda_{n} A\right) x-\left(I-\lambda_{n} A\right) y \|^{2} \\
= & \|x-y\|^{2}-2 \lambda_{n}\langle x-y, A x-A y\rangle \\
& +\lambda_{n}^{2}\|A x-A y\|^{2} \\
\leq & \|x-y\|^{2}-\lambda_{n}\left(2 \alpha-\lambda_{n}\right)\|A x-A y\|^{2} .
\end{aligned}
$$

It follows from restriction (a) that $I-\lambda_{n} A$ is nonexpansive.

Let $p \in \mathscr{F}$. It follows from (5), (16), and Lemmas 3 and 6 that

$$
\begin{aligned}
& \left\|x_{n+1}-p\right\|^{2} \\
& =\| P_{C}\left[\alpha_{n} u+\left(1-\alpha_{n}\right)\right. \\
& \times\left(\theta_{n} x_{n}+\delta_{n} T_{1} y_{n}+\gamma_{n} T_{2} z_{n}\right. \\
& \left.\left.+\xi_{n} J_{\lambda_{n}}\left(I-\lambda_{n} A\right) x_{n}\right)\right]-p \|^{2} \\
& \leq \| \alpha_{n} u+\left(1-\alpha_{n}\right) \\
& \times\left(\theta_{n} x_{n}+\delta_{n} T_{1} y_{n}+\gamma_{n} T_{2} z_{n}\right. \\
& \left.+\xi_{n} J_{\lambda_{n}}\left(I-\lambda_{n} A\right) x_{n}\right)-p \|^{2} \\
& \leq \alpha_{n}\|u-p\|^{2}+\left(1-\alpha_{n}\right) \\
& \times \| \theta_{n}\left(x_{n}-p\right)+\delta_{n}\left(T_{1} y_{n}-p\right) \\
& +\gamma_{n}\left(T_{2} z_{n}-p\right) \\
& +\xi_{n}\left(J_{\lambda_{n}}\left(I-\lambda_{n} A\right) x_{n}-p\right) \|^{2} \\
& \leq \alpha_{n}\|u-p\|^{2}+\left(1-\alpha_{n}\right) \\
& \times\left[\theta_{n}\left\|x_{n}-p\right\|^{2}+\delta_{n}\left\|T_{1} y_{n}-p\right\|^{2}\right. \\
& +\gamma_{n}\left\|T_{2} z_{n}-p\right\|^{2} \\
& \left.+\xi_{n}\left\|J_{\lambda_{n}}\left(I-\lambda_{n} A\right) x_{n}-p\right\|^{2}\right] \\
& -\left(1-\alpha_{n}\right) \theta_{n} \delta_{n}\left\|T_{1} y_{n}-x_{n}\right\|^{2} \\
& -\left(1-\alpha_{n}\right) \theta_{n} \gamma_{n}\left\|T_{2} z_{n}-x_{n}\right\|^{2} \\
& -\left(1-\alpha_{n}\right) \theta_{n} \xi_{n}\left\|J_{\lambda_{n}}\left(I-\lambda_{n} A\right) x_{n}-x_{n}\right\|^{2}
\end{aligned}
$$




$$
\begin{aligned}
\leq & \alpha_{n}\|u-p\|^{2}+\left(1-\alpha_{n}\right)\left(\theta_{n}+\xi_{n}\right)\left\|x_{n}-p\right\|^{2} \\
& +\left(1-\alpha_{n}\right) \delta_{n}\left[\left\|y_{n}-p\right\|^{2}+\left\|y_{n}-T_{1} y_{n}\right\|^{2}\right] \\
& +\left(1-\alpha_{n}\right) \gamma_{n}\left[\left\|z_{n}-p\right\|^{2}+\left\|z_{n}-T_{2} z_{n}\right\|^{2}\right] \\
& -\left(1-\alpha_{n}\right) \theta_{n} \delta_{n}\left\|T_{1} y_{n}-x_{n}\right\|^{2} \\
& -\left(1-\alpha_{n}\right) \theta_{n} \gamma_{n}\left\|T_{2} z_{n}-x_{n}\right\|^{2} \\
& -\left(1-\alpha_{n}\right) \theta_{n} \xi_{n}\left\|J_{\lambda_{n}}\left(I-\lambda_{n} A\right) x_{n}-x_{n}\right\|^{2} .
\end{aligned}
$$

It follows from (5), (16), and Lemma 6 that

$$
\begin{aligned}
\| z_{n}- & p \|^{2} \\
= & \left\|\left(1-c_{n}\right)\left(x_{n}-p\right)+c_{n}\left(T_{2} x_{n}-p\right)\right\|^{2} \\
= & \left(1-c_{n}\right)\left\|x_{n}-p\right\|^{2} \\
& +c_{n}\left\|T_{2} x_{n}-p\right\|^{2}-c_{n}\left(1-c_{n}\right)\left\|x_{n}-T_{2} x_{n}\right\|^{2} \\
\leq & \left(1-c_{n}\right)\left\|x_{n}-p\right\|^{2} \\
& +c_{n}\left[\left\|x_{n}-p\right\|+\left\|x_{n}-T_{2} x_{n}\right\|^{2}\right] \\
& -c_{n}\left(1-c_{n}\right)\left\|x_{n}-T_{2} x_{n}\right\|^{2} \\
= & \left\|x_{n}-p\right\|^{2}+c_{n}^{2}\left\|x_{n}-T_{2} x_{n}\right\|^{2}, \\
\| y_{n}- & p \|^{2} \\
= & \left\|\left(1-\beta_{n}\right)\left(x_{n}-p\right)+\beta_{n}\left(T_{1} x_{n}-p\right)\right\|^{2} \\
\leq & \left\|x_{n}-p\right\|^{2}+\beta_{n}^{2}\left\|x_{n}-T_{1} x_{n}\right\|^{2} .
\end{aligned}
$$

Similarly, we have that

$$
\begin{aligned}
\| y_{n}- & T_{1} y_{n} \|^{2} \\
= & \left\|\left(1-\beta_{n}\right)\left(x_{n}-T_{1} y_{n}\right)+\beta_{n}\left(T_{1} x_{n}-T_{1} y_{n}\right)\right\|^{2} \\
= & \left(1-\beta_{n}\right)\left\|x_{n}-T_{1} y_{n}\right\|^{2} \\
& +\beta_{n}\left\|T_{1} x_{n}-T_{1} y_{n}\right\|^{2} \\
& -\beta_{n}\left(1-\beta_{n}\right)\left\|x_{n}-T_{1} x_{n}\right\|^{2} \\
\leq & \left(1-\beta_{n}\right)\left\|x_{n}-T_{1} y_{n}\right\|^{2}+\beta_{n} L^{2}\left\|x_{n}-y_{n}\right\|^{2} \\
& -\beta_{n}\left(1-\beta_{n}\right)\left\|x_{n}-T_{1} x_{n}\right\|^{2} \\
= & \left(1-\beta_{n}\right)\left\|x_{n}-T_{1} y_{n}\right\|^{2} \\
& -\beta_{n}\left(1-\beta_{n}^{2} L^{2}-\beta_{n}\right)\left\|x_{n}-T_{1} x_{n}\right\|^{2} . \\
\| z_{n}- & T_{2} z_{n} \|^{2} \\
= & \left\|\left(1-c_{n}\right)\left(x_{n}-T_{2} z_{n}\right)+c_{n}\left(T_{2} x_{n}-T_{2} z_{n}\right)\right\|^{2}
\end{aligned}
$$

$$
\begin{aligned}
\leq & \left(1-c_{n}\right)\left\|x_{n}-T_{2} z_{n}\right\|^{2} \\
& -c_{n}\left(1-c_{n}^{2} L^{2}-c_{n}\right)\left\|x_{n}-T_{2} x_{n}\right\|^{2} .
\end{aligned}
$$

Substituting (19) and (20) into (18), we obtain that

$$
\begin{aligned}
& \left\|x_{n+1}-p\right\|^{2} \\
& \quad \leq \alpha_{n}\|u-p\|^{2} \\
& +\left(1-\alpha_{n}\right)\left(\theta_{n}+\xi_{n}\right)\left\|x_{n}-p\right\|^{2} \\
& +\left(1-\alpha_{n}\right) \delta_{n}\left[\left\|x_{n}-p\right\|^{2}+\beta_{n}^{2}\left\|x_{n}-T_{1} x_{n}\right\|^{2}\right] \\
& +\left(1-\alpha_{n}\right) \delta_{n}\left[\left(1-\beta_{n}\right)\left\|x_{n}-T_{1} y_{n}\right\|^{2}\right. \\
& +\left(1-\alpha_{n}\right) \gamma_{n}\left[\left\|x_{n}-p\right\|^{2}+c_{n}^{2}\left\|x_{n}-T_{2} x_{n}\right\|^{2}\right] \\
& +\left(1-\alpha_{n}\right) \gamma_{n}\left[\left(1-c_{n}\right)\left\|x_{n}-T_{2} z_{n}\right\|^{2}\right. \\
& +\left(1-\alpha_{n}\right) \theta_{n} \xi_{n}\left\|J_{\lambda_{n}}\left(I-\lambda_{n} A\right) x_{n}-x_{n}\right\|^{2} \cdot \\
& +\left(1-\alpha_{n}\right) \delta_{n}\left(\delta_{n}+\gamma_{n}+\xi_{n}-\beta_{n}\right)\left\|T_{1} y_{n}-x_{n}\right\|^{2} \\
& +\left(1-\alpha_{n}\right) \theta_{n} \delta_{n}\left\|T_{1} y_{n}-x_{n}\right\|^{2} \\
& + \\
& + \\
& + \\
& +
\end{aligned}
$$

In view of restriction $(\mathrm{d})$, we find that

$$
\begin{gathered}
1-2 \beta_{n}-\beta_{n}^{2} L^{2} \geq 1-2 \beta-\beta^{2} L^{2}>0, \\
1-2 c_{n}-c_{n}^{2} L^{2} \geq 1-2 \beta-\beta^{2} L^{2}>0, \\
\delta_{n}+\gamma_{n}+\xi_{n}-\beta_{n} \leq 0, \\
\delta_{n}+\gamma_{n}+\xi_{n}-c_{n} \leq 0,
\end{gathered}
$$

for all $n \geq 1$. It follows from (21) and (22) that

$$
\left\|x_{n+1}-p\right\|^{2} \leq \alpha_{n}\|u-p\|^{2}+\left(1-\alpha_{n}\right)\left\|x_{n}-p\right\|^{2} .
$$

Putting $M=\max \left\{\|u-p\|^{2},\left\|x_{1}-p\right\|^{2}\right\}$, we find that $\left\|x_{n}-p\right\|^{2} \leq M$ for all $n \geq 1$. 
Indeed, it is clear that $\left\|x_{2}-p\right\|^{2} \leq M$. Suppose that $\| x_{m}-$ $p \| \leq M$ for some positive integer $m$. It follows that

$$
\begin{aligned}
\left\|x_{m+1}-p\right\|^{2} & \leq \alpha_{m}\|u-p\|^{2}+\left(1-\alpha_{m}\right)\left\|x_{m}-p\right\|^{2} \\
& \leq \alpha_{m} M+\left(1-\alpha_{m}\right) M \\
& =M .
\end{aligned}
$$

This finds that $\left\{x_{n}\right\}$ is bounded and hence $\left\{y_{n}\right\}$ and $\left\{z_{n}\right\}$ are bounded.

Let $w_{n}=\theta_{n} x_{n}+\delta_{n} T_{1} y_{n}+\gamma_{n} T_{2} z_{n}+\xi_{n} J_{\lambda_{n}}\left(I-\lambda_{n} A\right) x_{n}$. Then we see that $x_{n+1}=P_{C}\left(\alpha_{n} u+\left(1-\alpha_{n}\right) w_{n}\right)$. Put $\bar{x}=P_{\mathscr{F}} u$. Using (16), (19), and (20) and Lemmas 1 and 6, we find that

$$
\begin{aligned}
&\left\|x_{n+1}-\bar{x}\right\|^{2} \\
& \quad \leq\left\|\alpha_{n}(u-\bar{x})+\left(1-\alpha_{n}\right)\left(w_{n}-\bar{x}\right)\right\|^{2} \\
& \leq\left(1-\alpha_{n}\right)\left\|w_{n}-\bar{x}\right\|^{2} \\
&+2 \alpha_{n}\left\langle u-\bar{x}, \alpha_{n}(u-\bar{x})+\left(1-\alpha_{n}\right)\left(w_{n}-\bar{x}\right)\right\rangle \\
& \leq\left(1-\alpha_{n}\right) \theta_{n}\left\|x_{n}-\bar{x}\right\|^{2} \\
&+\left(1-\alpha_{n}\right) \delta_{n}\left\|T_{1} y_{n}-\bar{x}\right\|^{2} \\
&+\left(1-\alpha_{n}\right) \gamma_{n}\left\|T_{2} z_{n}-\bar{x}\right\|^{2} \\
&+\left(1-\alpha_{n}\right) \xi_{n}\left\|J_{\lambda_{n}}\left(I-\lambda_{n} A\right) x_{n}-\bar{x}\right\|^{2} \\
&-\left(1-\alpha_{n}\right) \theta_{n} \delta_{n}\left\|T_{1} y_{n}-x_{n}\right\|^{2} \\
&-\left(1-\alpha_{n}\right) \theta_{n} \gamma_{n}\left\|T_{2} z_{n}-x_{n}\right\|^{2} \\
&-\left(1-\alpha_{n}\right) \theta_{n} \xi_{n}\left\|J_{\lambda_{n}}\left(I-\lambda_{n} A\right) x_{n}-x_{n}\right\|^{2} \\
&+2 \alpha_{n}^{2}\|u-\bar{x}\|^{2} \\
&+2 \alpha_{n}\left(1-\alpha_{n}\right)\left\langle u-\bar{x}, w_{n}-\bar{x}\right\rangle \\
& \leq\left(1-\alpha_{n}\right) \theta_{n}\left\|x_{n}-\bar{x}\right\|^{2} \\
&+\left(1-\alpha_{n}\right) \delta_{n}\left[\left\|y_{n}-\bar{x}\right\|^{2}+\left\|y_{n}-T_{1} y_{n}\right\|^{2}\right] \\
&+\left(1-\alpha_{n}\right) \gamma_{n}\left[\left\|z_{n}-\bar{x}\right\|^{2}+\left\|z_{n}-T_{2} z_{n}\right\|^{2}\right] \\
&+\left(1-\alpha_{n}^{2}\|u-\bar{x}\|^{2}\right. \\
&-\left(1-\alpha_{n}\right) \theta_{n} \delta_{n}\left\|T_{1} y_{n}-x_{n}\right\|^{2} \\
&-\left(1-\alpha_{n}\right) \theta_{n} \gamma_{n}\left\|T_{2} z_{n}-x_{n}\right\|^{2} \\
&+J_{\lambda_{n}}\left(I-\lambda_{n} A\right) x_{n}-x_{n} \|^{2} \\
&\left(1-\alpha_{n}\right.
\end{aligned}
$$

$$
\begin{aligned}
& +2 \alpha_{n}\left(1-\alpha_{n}\right)\left\langle u-\bar{x}, w_{n}-\bar{x}\right\rangle \\
& \leq\left(1-\alpha_{n}\right)\left(\theta_{n}+\xi_{n}\right)\left\|x_{n}-\bar{x}\right\|^{2} \\
& +\left(1-\alpha_{n}\right) \delta_{n}\left[\left\|x_{n}-\bar{x}\right\|^{2}+\beta_{n}^{2}\left\|x_{n}-T_{1} x_{n}\right\|^{2}\right] \\
& +\left(1-\alpha_{n}\right) \delta_{n}\left[\left(1-\beta_{n}\right)\left\|x_{n}-T_{1} y_{n}\right\|^{2}\right. \\
& \left.\quad-\beta_{n}\left(1-\beta_{n}^{2} L^{2}-\beta_{n}\right)\left\|x_{n}-T_{1} x_{n}\right\|^{2}\right] \\
& +\left(1-\alpha_{n}\right) \gamma_{n}\left[\left\|x_{n}-\bar{x}\right\|^{2}+c_{n}^{2}\left\|x_{n}-T_{2} x_{n}\right\|^{2}\right] \\
& +\left(1-\alpha_{n}\right) \gamma_{n}\left[\left(1-c_{n}\right)\left\|x_{n}-T_{2} z_{n}\right\|^{2}\right. \\
& \left.\quad-c_{n}\left(1-c_{n}^{2} L^{2}-c_{n}\right)\left\|x_{n}-T_{2} x_{n}\right\|^{2}\right] \\
& -\left(1-\alpha_{n}\right) \theta_{n} \delta_{n}\left\|T_{1} y_{n}-x_{n}\right\|^{2} \\
& -\left(1-\alpha_{n}\right) \theta_{n} \gamma_{n}\left\|T_{2} z_{n}-x_{n}\right\|^{2} \\
& -\left(1-\alpha_{n}\right) \theta_{n} \xi_{n}\left\|J_{\lambda_{n}}\left(I-\lambda_{n} A\right) x_{n}-x_{n}\right\|^{2} \\
& +2 \alpha_{n}^{2}\|u-\bar{x}\|^{2}+2 \alpha_{n}\left(1-\alpha_{n}\right)\left\langle u-\bar{x}, w_{n}-\bar{x}\right\rangle,
\end{aligned}
$$

which implies from (22) that

$$
\begin{aligned}
& \left\|x_{n+1}-\bar{x}\right\|^{2} \\
& \quad \leq\left(1-\alpha_{n}\right)\left\|x_{n}-\bar{x}\right\|^{2} \\
& \quad-\left(1-\alpha_{n}\right) \delta_{n} \beta_{n}\left(1-2 \beta_{n}-\beta_{n}^{2} L^{2}\right)\left\|x_{n}-T_{1} x_{n}\right\|^{2} \\
& \quad+\left(1-\alpha_{n}\right) \delta_{n}\left(\delta_{n}+\xi_{n}+\gamma_{n}-\beta_{n}\right)\left\|T_{1} y_{n}-x_{n}\right\|^{2} \\
& \quad-\left(1-\alpha_{n}\right) \gamma_{n} c_{n}\left(1-2 c_{n}-c_{n}^{2} L^{2}\right)\left\|x_{n}-T_{2} x_{n}\right\|^{2} \\
& \quad+\left(1-\alpha_{n}\right) \gamma_{n}\left(\delta_{n}+\xi_{n}+\gamma_{n}-c_{n}\right)\left\|T_{2} z_{n}-x_{n}\right\| \\
& \quad-\left(1-\alpha_{n}\right) \theta_{n} \xi_{n}\left\|J_{\lambda_{n}}\left(I-\lambda_{n} A\right) x_{n}-x_{n}\right\|^{2} \\
& \quad+2 \alpha_{n}^{2}\|u-\bar{x}\|^{2}+2 \alpha_{n}\left(1-\alpha_{n}\right)\left\langle u-\bar{x}, w_{n}-\bar{x}\right\rangle \\
& \leq \\
& +\left(1-\alpha_{n}\right)\left\|x_{n}-\bar{x}\right\|^{2}+2 \alpha_{n}^{2}\|u-\bar{x}\|^{2} \\
& \quad+2 \alpha_{n}\left(1-\alpha_{n}\right)\left\langle u-\bar{x}, w_{n}-\bar{x}\right\rangle .
\end{aligned}
$$

Now we consider two cases.

Case 1. Suppose that there exists $n_{0} \in N$ such that $\left\{\left\|x_{n}-\bar{x}\right\|\right\}$ is decreasing for all $n \geq n_{0}$. Then we get that $\left\{\left\|x_{n}-\bar{x}\right\|\right\}$ is convergent. It follows from (22) and (26) that

$$
\begin{gathered}
x_{n}-T_{1} x_{n} \longrightarrow 0, \quad x_{n}-T_{2} x_{n} \longrightarrow 0, \\
x_{n}-J_{\lambda_{n}}\left(I-\lambda_{n} A\right) x_{n} \longrightarrow 0,
\end{gathered}
$$

as $n \rightarrow \infty$. Also we obtain from (27) that

$$
\begin{aligned}
& \left\|y_{n}-x_{n}\right\|=\beta_{n}\left\|x_{n}-T_{1} x_{n}\right\| \longrightarrow 0, \\
& \left\|z_{n}-x_{n}\right\|=c_{n}\left\|x_{n}-T_{2} x_{n}\right\| \longrightarrow 0,
\end{aligned}
$$


as $n \rightarrow \infty$. In view of the Lipschitz continuity of $T_{1}, T_{2}$ and (27) and (28), we find that

$$
\begin{aligned}
\left\|T_{1} y_{n}-x_{n}\right\| & \leq\left\|T_{1} y_{n}-T_{1} x_{n}\right\|+\left\|T_{1} x_{n}-x_{n}\right\| \\
& \leq L\left\|y_{n}-x_{n}\right\|+\left\|T_{1} x_{n}-x_{n}\right\| \\
& \longrightarrow 0 \text { as } n \longrightarrow \infty, \\
\left\|T_{2} z_{n}-x_{n}\right\| & \leq\left\|T_{2} z_{n}-T_{2} x_{n}\right\|+\left\|T_{2} x_{n}-x_{n}\right\| \\
& \leq L\left\|z_{n}-x_{n}\right\|+\left\|T_{2} x_{n}-x_{n}\right\| \\
& \longrightarrow 0 \text { as } n \longrightarrow \infty .
\end{aligned}
$$

It follows from (27), (29), and (30) that

$$
\begin{aligned}
\left\|w_{n}-x_{n}\right\| \leq & \delta_{n}\left\|T_{1} y_{n}-x_{n}\right\|+\gamma_{n}\left\|T_{2} z_{n}-x_{n}\right\| \\
& +\xi_{n}\left\|J_{\lambda_{n}}\left(I-\lambda_{n} A\right) x_{n}-x_{n}\right\| \\
& \longrightarrow 0 \text { as } n \longrightarrow \infty .
\end{aligned}
$$

Since $\left\{w_{n}\right\}$ is a bounded subset of $H$, we can choose a subsequence $\left\{w_{n_{i}}\right\}$ of $\left\{w_{n}\right\}$ such that $w_{n_{i}} \rightarrow w$ and

$$
\limsup _{n \rightarrow \infty}\left\langle u-\bar{x}, w_{n}-\bar{x}\right\rangle=\lim _{i \rightarrow \infty}\left\langle u-\bar{x}, w_{n_{i}}-\bar{x}\right\rangle .
$$

It follows from (31) that $x_{n_{i}} \rightarrow w$. By (27) and Lemma 7, we obtain that $w \in F\left(T_{1}\right)$ and $w \in F\left(T_{2}\right)$.

Next, we show that $w \in(A+B)^{-1} 0$.

Notice that

$$
\begin{aligned}
&\left\|J_{\lambda_{n}}\left(I-\lambda_{n} A\right) x_{n}-p\right\|^{2} \\
&=\left\|J_{\lambda_{n}}\left(I-\lambda_{n} A\right) x_{n}-J_{\lambda_{n}}\left(I-\lambda_{n} A\right) p\right\|^{2} \\
& \leq\left\|\left(I-\lambda_{n} A\right) x_{n}-\left(I-\lambda_{n} A\right) p\right\|^{2} \\
&=\left\|x_{n}-p\right\|^{2}-2 \lambda_{n}\left\langle x_{n}-p, A x_{n}-A p\right\rangle \\
& \quad+\lambda_{n}^{2}\left\|A x_{n}-A p\right\|^{2} \\
& \leq\left\|x_{n}-p\right\|^{2}-2 \alpha \lambda_{n}\left\|A x_{n}-A p\right\|^{2}+\lambda_{n}^{2}\left\|A x_{n}-A p\right\|^{2} \\
&=\left\|x_{n}-p\right\|^{2}-\lambda_{n}\left(2 \alpha-\lambda_{n}\right)\left\|A x_{n}-A p\right\|^{2} .
\end{aligned}
$$

It follows from (27) that

$$
\begin{aligned}
\lambda_{n}\left(2 \alpha-\lambda_{n}\right)\left\|A x_{n}-A p\right\|^{2} \\
\leq\left\|x_{n}-p\right\|^{2}-\left\|J_{\lambda_{n}}\left(I-\lambda_{n} A\right) x_{n}-p\right\| \\
=\left(\left\|x_{n}-p\right\|+\left\|J_{\lambda_{n}}\left(I-\lambda_{n} A\right) x_{n}-p\right\|\right) \\
\quad \times\left\|x_{n}-J_{\lambda_{n}}\left(I-\lambda_{n} A\right) x_{n}\right\| \\
\quad \longrightarrow 0 \text { as } n \longrightarrow \infty .
\end{aligned}
$$

Hence we get

$$
\lim _{n \rightarrow \infty}\left\|A x_{n}-A p\right\|^{2}=0
$$

Putting $h_{n}=J_{\lambda_{n}}\left(I-\lambda_{n} A\right) x_{n}$, we find that $\left(\left(x_{n_{i}}-h_{n_{i}}\right) / \lambda_{n_{i}}\right)-$ $A x_{n_{i}} \in B h_{n_{i}}$. Since $B$ is monotone, we get that, for any $(u, v) \in$ $G(B)$,

$$
\left\langle h_{n_{i}}-u, \frac{x_{n_{i}}-h_{n_{i}}}{\lambda_{n_{i}}}-A x_{n_{i}}-v\right\rangle \geq 0,
$$

where $G(B)=\{(x, w) \in H \times H: x \in D(B), w \in B x\}$. Since $\left\langle x_{n_{i}}-w, A x_{n_{i}}-A w\right\rangle \geq \alpha\left\|A x_{n_{i}}-A w\right\|^{2}, x_{n_{i}} \rightarrow w$, and $A x_{n_{i}} \rightarrow$ $A p$ as $i \rightarrow \infty$, we have $A x_{n_{i}} \rightarrow A w$. Thus, letting $i \rightarrow \infty$, we obtain from (27) and (36) that $\langle w-u,-A w-v\rangle \geq 0$. This means $-A w \in B w$, that is, $0 \in(A+B) w$. Hence we get $w \in(A+B)^{-1} 0$. This implies from Lemma 2 that

$$
\begin{aligned}
\limsup _{n \rightarrow \infty}\left\langle u-\bar{x}, w_{n}-\bar{x}\right\rangle & =\lim _{i \rightarrow \infty}\left\langle u-\bar{x}, w_{n_{i}}-\bar{x}\right\rangle \\
& =\langle u-\bar{x}, w-\bar{x}\rangle \\
& \leq 0 .
\end{aligned}
$$

On the other hand, we have from (26) that

$$
\begin{aligned}
\left\|x_{n+1}-\bar{x}\right\|^{2} \leq & \left(1-\alpha_{n}\right)\left\|x_{n}-\bar{x}\right\|^{2} \\
& +\alpha_{n}\left(2 \alpha_{n}\|u-\bar{x}\|^{2}\right. \\
& \left.+\left(1-\alpha_{n}\right)\left\langle u-\bar{x}, w_{n}-\bar{x}\right\rangle\right) .
\end{aligned}
$$

From Lemma 8 and (37), we find that $\lim _{n \rightarrow \infty}\left\|x_{n}-\bar{x}\right\|=0$.

Case 2. Suppose that there exists a subsequence $\left\{n_{i}\right\}$ of $\{n\}$ such that

$$
\left\|x_{n_{i}}-\bar{x}\right\|<\left\|x_{n_{i}+1}-\bar{x}\right\|
$$

for all $i \in \mathbb{N}$. By Lemma 5, there exists a nondecreasing sequence $\left\{m_{k}\right\} \subset \mathbb{N}$ such that $m_{k} \rightarrow \infty$ and

$$
\left\|x_{m_{k}}-\bar{x}\right\| \leq\left\|x_{m_{k}+1}-\bar{x}\right\|, \quad\left\|x_{k}-\bar{x}\right\| \leq\left\|x_{m_{k}+1}-\bar{x}\right\|,
$$

for all $k \in \mathbb{N}$. From (22) and (26), we have $x_{m_{k}}-T_{1} x_{m_{k}} \rightarrow 0$, $x_{m_{k}}-T_{2} x_{m_{k}} \rightarrow 0$, and $x_{m_{k}}-J_{\lambda_{m_{k}}}\left(I-\lambda_{m_{k}} A\right) x_{m_{k}} \stackrel{\rightarrow}{\rightarrow} 0$ as $k \rightarrow \infty$. Thus, like in Case 1 , we obtain $w_{m_{k}}-x_{m_{k}} \rightarrow 0$ and

$$
\limsup _{k \rightarrow \infty}\left\langle u-\bar{x}, w_{m_{k}}-\bar{x}\right\rangle \leq 0
$$

From (26) and (40), we have

$$
\begin{aligned}
\alpha_{m_{k}} & \left\|x_{m_{k}}-\bar{x}\right\|^{2} \\
\leq & \left\|x_{m_{k}}-\bar{x}\right\|^{2}-\left\|x_{m_{k}+1}-\bar{x}\right\|^{2} \\
& +2 \alpha_{m_{k}}\left(\alpha_{m_{k}}\|u-\bar{x}\|^{2}+\left(1-\alpha_{m_{k}}\right)\left\langle u-\bar{x}, w_{m_{k}}-\bar{x}\right\rangle\right) \\
\leq & 2 \alpha_{m_{k}}\left(\alpha_{m_{k}}\|u-\bar{x}\|^{2}+\left(1-\alpha_{m_{k}}\right)\left\langle u-\bar{x}, w_{m_{k}}-\bar{x}\right\rangle\right) .
\end{aligned}
$$


Applying (41) and $\alpha_{m_{k}}>0$, we have $\left\|x_{m_{k}}-\bar{x}\right\| \rightarrow 0$ as $k \rightarrow$ $\infty$. It implies that $\left\|x_{m_{k}+1}-\bar{x}\right\| \rightarrow 0$ as $k \rightarrow \infty$. By (40), we have $x_{k} \rightarrow \bar{x}$ as $k \rightarrow \infty$.

Therefore, from the above two cases, we can conclude that the sequence $\left\{x_{n}\right\}$ converges strongly to $\bar{x}=P_{\mathscr{F}} u$. This completes the proof.

From Lemma 4, we have the following result.

Corollary 10. Let $C$ be a nonempty closed convex subset of a real Hilbert space $H$ such that int $(C) \neq \phi$. Let $T_{1}, T_{2}: C \rightarrow$ $C$ be Lipschitzian pseudocontractive mappings with Lipschitz constants $L_{1}$ and $L_{2}$, respectively. Let $B_{1}: D\left(B_{1}\right) \rightarrow 2^{H}$ and $B_{2}: D\left(B_{2}\right) \rightarrow 2^{H}$ be maximal monotone mappings such that $D\left(B_{1}\right) \cap \operatorname{int}\left(D\left(B_{2}\right)\right) \neq \phi$. Assume that $\mathscr{F}=F\left(T_{1}\right) \cap F\left(T_{2}\right) \cap$ $\left(B_{1}+B_{2}\right)^{-1}(0) \neq \phi$. Let $J_{\lambda_{n}}=\left(I+\lambda_{n}\left(B_{1}+B_{2}\right)\right)^{-1}$, where $\left\{\lambda_{n}\right\}$ is a positive real number sequence. Given $x_{1}, u \in C$, let $\left\{x_{n}\right\}$ be the sequence generated by the following algorithm:

$$
\begin{gathered}
z_{n}=\left(1-c_{n}\right) x_{n}+c_{n} T_{2} x_{n}, \\
y_{n}=\left(1-\beta_{n}\right) x_{n}+\beta_{n} T_{1} x_{n}, \\
x_{n+1}=P_{C}\left[\alpha_{n} u+\left(1-\alpha_{n}\right)\right. \\
\left.\times\left(\theta_{n} x_{n}+\delta_{n} T_{1} y_{n}+\gamma_{n} T_{2} z_{n}+\xi_{n} J_{\lambda_{n}} x_{n}\right)\right], \\
\forall n \geq 1 .
\end{gathered}
$$

Assume that the sequences $\left\{\alpha_{n}\right\},\left\{\beta_{n}\right\},\left\{c_{n}\right\},\left\{\theta_{n}\right\},\left\{\delta_{n}\right\},\left\{\gamma_{n}\right\},\left\{\xi_{n}\right\}$, and $\left\{\lambda_{n}\right\}$ satisfy the following restrictions:
(a) $0<a<\lambda_{n}<b<1$;
(b) $0<c \leq \theta_{n}, \delta_{n}, \gamma_{n}, \xi_{n} \leq d<1$, and $\theta_{n}+\delta_{n}+\gamma_{n}+\xi_{n}=1$;
(c) $0<\alpha_{n}<e<1, \lim _{n \rightarrow \infty} \alpha_{n}=0$, and $\sum_{n=1}^{\infty} \alpha_{n}=\infty$;
(d) $\delta_{n}+\gamma_{n}+\xi_{n} \leq \beta_{n}, c_{n} \leq \beta<1 /\left(\sqrt{1+L^{2}}+1\right)$, for all $n \geq 1$,

for some real numbers $a, b, c, d, e>0$, where $L=\max \left\{L_{1}, L_{2}\right\}$. Then $\left\{x_{n}\right\}$ converges strongly to some point $\bar{x}$, where $\bar{x}=P_{\mathscr{F}} u$.

Remark 11. If $T_{1}=T, T_{2}=I$ (the identity mapping), and $u=0$, then Theorem 9 reduces to Theorem 3.1 of Shahzad and Zegeye [6]. Thus, Theorem 9 covers Theorem 3.1 of Shahzad and Zegeye [6] as a special case.

\section{Applications}

In this section, we will consider equilibrium problems and variational inequalities.

Let $C$ be a nonempty closed convex subset of a real Hilbert space $H$. Let $F$ be a bifunction of $C \times C$ into $\mathbb{R}$, where $\mathbb{R}$ denotes the set of real numbers. Recall the following equilibrium problem: find $x \in C$ such that

$$
F(x, y) \geq 0, \quad \forall y \in C .
$$

We use $\operatorname{EP}(F)$ to denote the solution set of the equilibrium problem. To study the equilibrium problems, we assume that $F$ satisfies the following conditions:
(A1) $F(x, x)=0$ for all $x \in C$;

(A2) $F$ is monotone, that is, $F(x, y)+F(y, x) \geq 0$, for all $x, y \in C$;

(A3) for each $x, y, z \in C$,

$$
\limsup _{t \downarrow 0} F(t z+(1-t) x, y) \leq F(x, y)
$$

(A4) for each $x \in C, y \mapsto F(x, y)$ is convex and lower semicontinuous.

Lemma 12 (see [1]). Let $C$ be a nonempty closed convex subset of a real Hilbert space and let $F: C \times C \rightarrow \mathbb{R}$ be a bifunction satisfying (A1)-(A4). Then, for any $r>0$ and $x \in H$, there exists $z \in C$ such that

$$
F(z, y)+\frac{1}{r}\langle y-z, z-x\rangle \geq 0, \quad \forall y \in C
$$

Further, define

$$
T_{r}(x)=\left\{z \in C: F(z, y)+\frac{1}{r}\langle y-z, z-x\rangle \geq 0, \forall y \in C\right\},
$$

for all $r>0$ and $x \in H$. Then the following hold:

(a) $T_{r}$ is single-valued;

(b) $T_{r}$ is firmly nonexpansive; that is, for any $x, y \in H$,

$$
\left\|T_{r} x-T_{r} y\right\|^{2} \leq\left\langle T_{r} x-T_{r} y, x-y\right\rangle
$$

(c) $F\left(T_{r}\right)=\mathrm{EP}(F)$;

(d) $\mathrm{EP}(F)$ is closed and convex.

Lemma 13 (see [13]). Let $C$ be a nonempty closed convex subset of a real Hilbert space $H$, let $F$ be a bifunction from $C \times C$ to $\mathbb{R}$ which satisfies (A1)-(A4), and let $A_{F}$ be a multivalued mapping of $H$ into itself defined by

$$
A_{F} x= \begin{cases}\{z \in H: F(x, y) \geq\langle y-x, z\rangle, \forall y \in C\}, & x \in C, \\ \phi, & x \notin C .\end{cases}
$$

Then $A_{F}$ is a maximal monotone mapping with the domain $D\left(A_{F}\right) \subset C, \operatorname{EP}(F)=A_{F}^{-1} 0$, and

$$
T_{r}(x)=\left(I+r A_{F}\right)^{-1} x, \quad \forall x \in H, r>0,
$$

where $T_{r}$ is defined as in (47).

Now we consider an equilibrium problem. Using Lemmas 12 and 13, the following result holds.

Theorem 14. Let $C$ be a nonempty closed convex subset of a real Hilbert space $H$. Let $T_{1}, T_{2}: C \rightarrow C$ be Lipschitzian pseudocontractive mappings with Lipschitz constants $L_{1}$ and $L_{2}$, respectively. Assume that $\mathscr{F}=F\left(T_{1}\right) \cap F\left(T_{2}\right) \cap \mathrm{EP}(F) \neq \phi$. 
Given $x_{1}, u \in C$, let $\left\{x_{n}\right\}$ be the sequence generated by the following algorithm:

$$
\begin{gathered}
z_{n}=\left(1-c_{n}\right) x_{n}+c_{n} T_{2} x_{n}, \\
y_{n}=\left(1-\beta_{n}\right) x_{n}+\beta_{n} T_{1} x_{n}, \\
u_{n} \in C \quad \text { such that } F\left(u_{n}, v\right)+\frac{1}{r_{n}}\left\langle v-u_{n}, u_{n}-x_{n}\right\rangle \geq 0, \\
\quad \forall v \in C, \\
x_{n+1}=P_{C}\left[\alpha_{n} u+\left(1-\alpha_{n}\right)\right. \\
\left.\times\left(\theta_{n} x_{n}+\delta_{n} T_{1} y_{n}+\gamma_{n} T_{2} z_{n}+\xi_{n} u_{n}\right)\right], \\
\forall n \geq 1 .
\end{gathered}
$$

Assume that the sequences $\left\{\alpha_{n}\right\},\left\{\beta_{n}\right\},\left\{c_{n}\right\},\left\{\theta_{n}\right\},\left\{\delta_{n}\right\},\left\{\gamma_{n}\right\},\left\{\xi_{n}\right\}$, and $\left\{r_{n}\right\}$ satisfy the following restrictions:

(a) $\liminf _{n \rightarrow \infty} r_{n}>0$ and $\lim _{n \rightarrow \infty}\left\|r_{n+1}-r_{n}\right\|=0$;

(b) $0<c \leq \theta_{n}, \delta_{n}, \gamma_{n}, \xi_{n} \leq d<1$, and $\theta_{n}+\delta_{n}+\gamma_{n}+\xi_{n}=1$;

(c) $0<\alpha_{n}<e<1, \lim _{n \rightarrow \infty} \alpha_{n}=0$, and $\sum_{n=1}^{\infty} \alpha_{n}=\infty$;

(d) $\delta_{n}+\gamma_{n}+\xi_{n} \leq \beta_{n}, c_{n}<\beta<1 /\left(\sqrt{1+L^{2}}+1\right)$, for all $n \geq 1$,

for some real numbers $c, d, e>0$, where $L=\max \left\{L_{1}, L_{2}\right\}$. Then $\left\{x_{n}\right\}$ converges strongly to some point $\bar{x}$, where $\bar{x}=P_{\mathscr{F}} u$.

Let $f: H \rightarrow(-\infty,+\infty]$ be a proper convex lower semicontinuous function. Then the subdifferential of $\partial f$ of $f$ is defined as follows:

$$
\begin{array}{r}
\partial f(x)=\{y \in H: f(z) \geq f(x)+\langle z-x, y\rangle, \quad z \in H\}, \\
\forall x \in H .
\end{array}
$$

From Rockafellar [14], we find that $\partial f$ is maximal monotone. It is easy to verify that $0 \in \partial f(x)$ if and only if $f(x)=$ $\min _{y \in H} f(y)$. Let $I_{C}$ be the indicator function of $C$; that is,

$$
I_{C}(x)= \begin{cases}0, & x \in C, \\ +\infty, & x \notin C .\end{cases}
$$

Then $I_{C}: H \rightarrow(-\infty,+\infty]$ is a proper convex lower semicontinuous function and $\partial I_{C}$ is a maximal monotone mapping.

Lemma 15 (see [6]). Let $C$ be a nonempty closed convex subset of a real Hilbert space $H$, let $P_{C}$ be the metric projection from $H$ onto $C$, and let $\partial I_{C}$ be the subdifferential of $I_{C}$, where $I_{C}$ is the indicator function of $C$ and let $J_{\lambda}=\left(I+\lambda \partial I_{C}\right)^{-1}$. Then

$$
y=J_{\lambda} x \Longleftrightarrow y=P_{C} x, \quad x \in H, y \in C .
$$

Now we consider a variational inequality problem.

Theorem 16. Let $C$ be a nonempty closed convex subset of a real Hilbert space $H$. Let $T_{1}, T_{2}: C \rightarrow C$ be Lipschitzian pseudocontractive mappings with Lipschitz constants $L_{1}$ and $L_{2}$, respectively. Let $A: C \rightarrow H$ be an $\alpha$-inverse strongly monotone mapping. Assume that $F\left(T_{1}\right) \cap F\left(T_{2}\right) \cap \operatorname{VI}(C, A) \neq \phi$. Given $x_{1}, u \in C$, let $\left\{x_{n}\right\}$ be the sequence generated by the following algorithm:

$$
\begin{gathered}
z_{n}=\left(1-c_{n}\right) x_{n}+c_{n} T_{2} x_{n}, \\
y_{n}=\left(1-\beta_{n}\right) x_{n}+\beta_{n} T_{1} x_{n}, \\
x_{n+1}=P_{C}\left[\alpha_{n} u+\left(1-\alpha_{n}\right)\right. \\
\times\left(\theta_{n} x_{n}+\delta_{n} T_{1} y_{n}+\gamma_{n} T_{2} z_{n}\right. \\
\left.\left.\quad+\xi_{n} P_{C}\left(I-\lambda_{n} A\right) x_{n}\right)\right], \quad \forall n \geq 1 .
\end{gathered}
$$

Assume that the sequences $\left\{\alpha_{n}\right\},\left\{\beta_{n}\right\},\left\{c_{n}\right\},\left\{\theta_{n}\right\},\left\{\delta_{n}\right\},\left\{\gamma_{n}\right\},\left\{\xi_{n}\right\}$, and $\left\{\lambda_{n}\right\}$ satisfy the following restrictions:

(a) $0<a<\lambda_{n}<b<2 \alpha$;

(b) $0<c \leq \theta_{n}, \delta_{n}, \gamma_{n}, \xi_{n} \leq d<1$, and $\theta_{n}+\delta_{n}+\gamma_{n}+\xi_{n}=1$;

(c) $0<\alpha_{n}<e<1, \lim _{n \rightarrow \infty} \alpha_{n}=0$, and $\sum_{n=1}^{\infty} \alpha_{n}=\infty$;

(d) $\delta_{n}+\gamma_{n}+\xi_{n} \leq \beta_{n}, c_{n}<\beta<1 /\left(\sqrt{1+L^{2}}+1\right)$, for all $n \geq 1$,

for some real numbers $a, b, c, d, e>0$, where $L=\max \left\{L_{1}, L_{2}\right\}$. Then $\left\{x_{n}\right\}$ converges strongly to some point $\bar{x}$, where $\bar{x}=P_{\mathscr{F}} u$.

Proof. Put $B=\partial I_{C}$ in Theorem 9. Then we get that

$$
\begin{aligned}
x \in\left(A+\partial I_{C}\right)^{-1} 0 & \Longleftrightarrow 0 \in A x+\partial I_{C} x \\
& \Longleftrightarrow-A x \in \partial I_{C} x \\
& \Longleftrightarrow\langle A x, y-x\rangle \geq 0 \\
& \Longleftrightarrow x \in \mathrm{VI}(C, A) .
\end{aligned}
$$

From Lemma 15, we can conclude the desired conclusion immediately.

\section{Conflict of Interests}

The author declares that there is no conflict of interests regarding the publication of this paper.

\section{References}

[1] E. Blum and W. Oettli, "From optimization and variational inequalities to equilibrium problems," The Mathematics Student, vol. 63, no. 1-4, pp. 123-145, 1994.

[2] S. Y. Cho, "Strong convergence of an iterative algorithm for sums of two monotone operators," Journal of Fixed Point Theory, vol. 2013, article 6, 2013.

[3] O. Güler, "On the convergence of the proximal point algorithm for convex minimization," SIAM Journal on Control and Optimization, vol. 29, no. 2, pp. 403-419, 1991.

[4] P.-E. Maingé, "Strong convergence of projected subgradient methods for nonsmooth and nonstrictly convex minimization," Set-Valued Analysis, vol. 16, no. 7-8, pp. 899-912, 2008. 
[5] N. Shahzad and H. Zegeye, "Approximating a common point of fixed points of a pseudocontractive mapping and zeros of sum of monotone mappings," Fixed Point Theory and Applications, vol. 2014, article 85, 2014.

[6] S. Takahashi, W. Takahashi, and M. Toyoda, "Strong convergence theorems for maximal monotone operators with nonlinear mappings in Hilbert spaces," Journal of Optimization Theory and Applications, vol. 147, no. 1, pp. 27-41, 2010.

[7] Q.-b. Zhang and C.-z. Cheng, "Strong convergence theorem for a family of Lipschitz pseudocontractive mappings in a Hilbert space," Mathematical and Computer Modelling, vol. 48, no. 3-4, pp. $480-485,2008$.

[8] R. T. Rockafellar, "Augmented Lagrangians and applications of the proximal point algorithm in convex programming," Mathematics of Operations Research, vol. 1, no. 2, pp. 97-116, 1976.

[9] R. T. Rockafellar, "Monotone operators and the proximal point algorithm," SIAM Journal on Control and Optimization, vol. 14, no. 5, pp. 877-898, 1976.

[10] Y. Alber, "Metric and generalized projection operators in Banach spaces: properties and applications," in Theory and Applications of Nonlinear Operators of Accretive and Monotone Type, A. G. Kartsatos, Ed., vol. 178 of Lecture Notes in Pure and Applied Mathematics, pp. 15-50, Marcel Dekker, New York, NY, USA, 1996.

[11] R. T. Rockafellar, "On the maximality of sums of nonlinear monotone operators," Transactions of the American Mathematical Society, vol. 149, pp. 75-88, 1970.

[12] H. Zegeye and N. Shahzad, “Convergence of Mann's type iteration method for generalized asymptotically nonexpansive mappings," Computers \& Mathematics with Applications, vol. 62, no. 11, pp. 4007-4014, 2011.

[13] H.-K. Xu, "Another control condition in an iterative method for nonexpansive mappings," Bulletin of the Australian Mathematical Society, vol. 65, no. 1, pp. 109-113, 2002.

[14] R. T. Rockafellar, "Characterization of the subdifferentials of convex functions," Pacific Journal of Mathematics, vol. 17, pp. 497-510, 1966. 


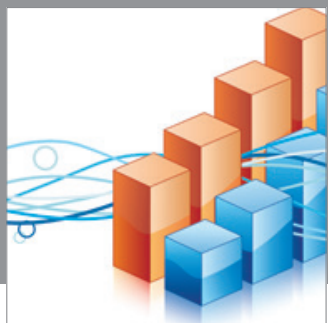

Advances in

Operations Research

mansans

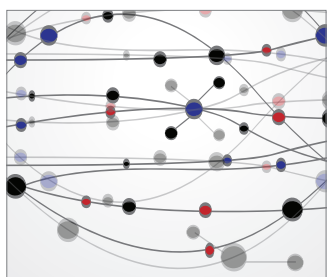

The Scientific World Journal
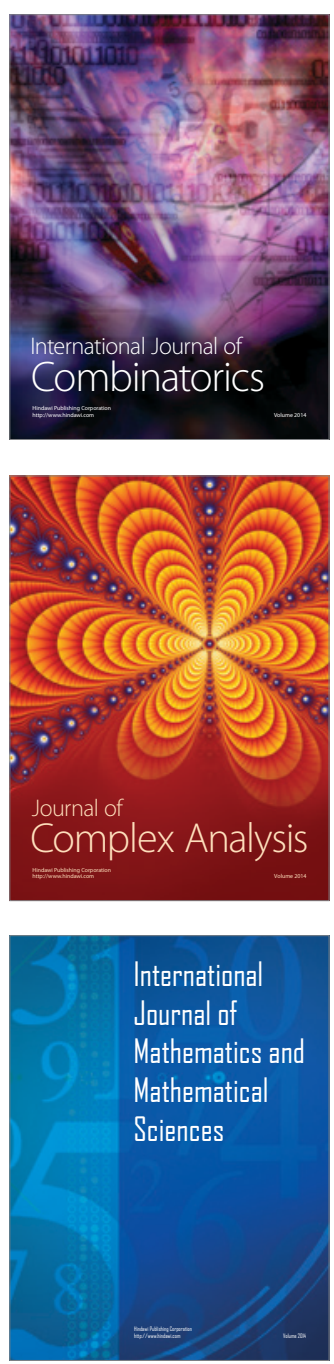
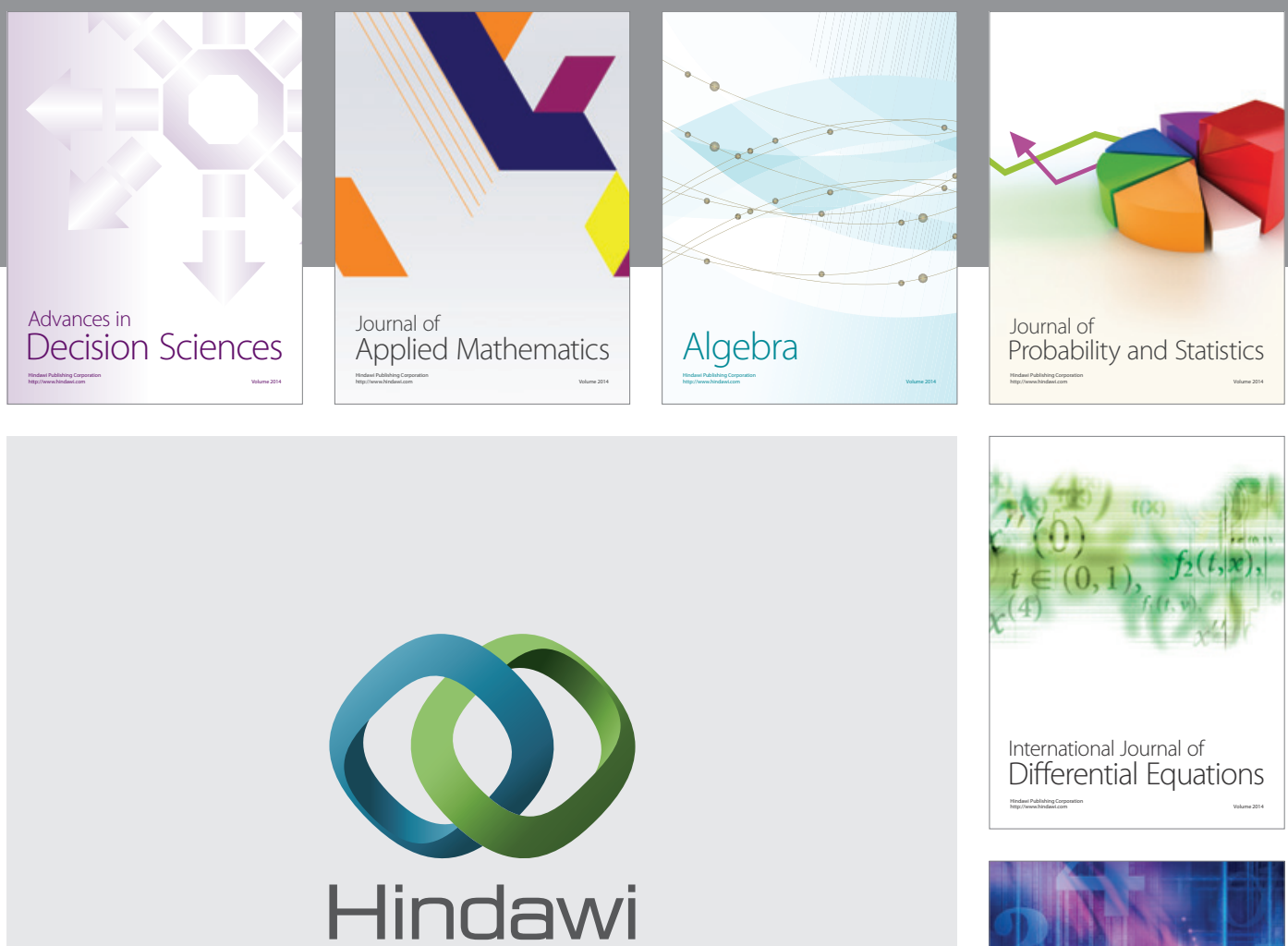

Submit your manuscripts at http://www.hindawi.com
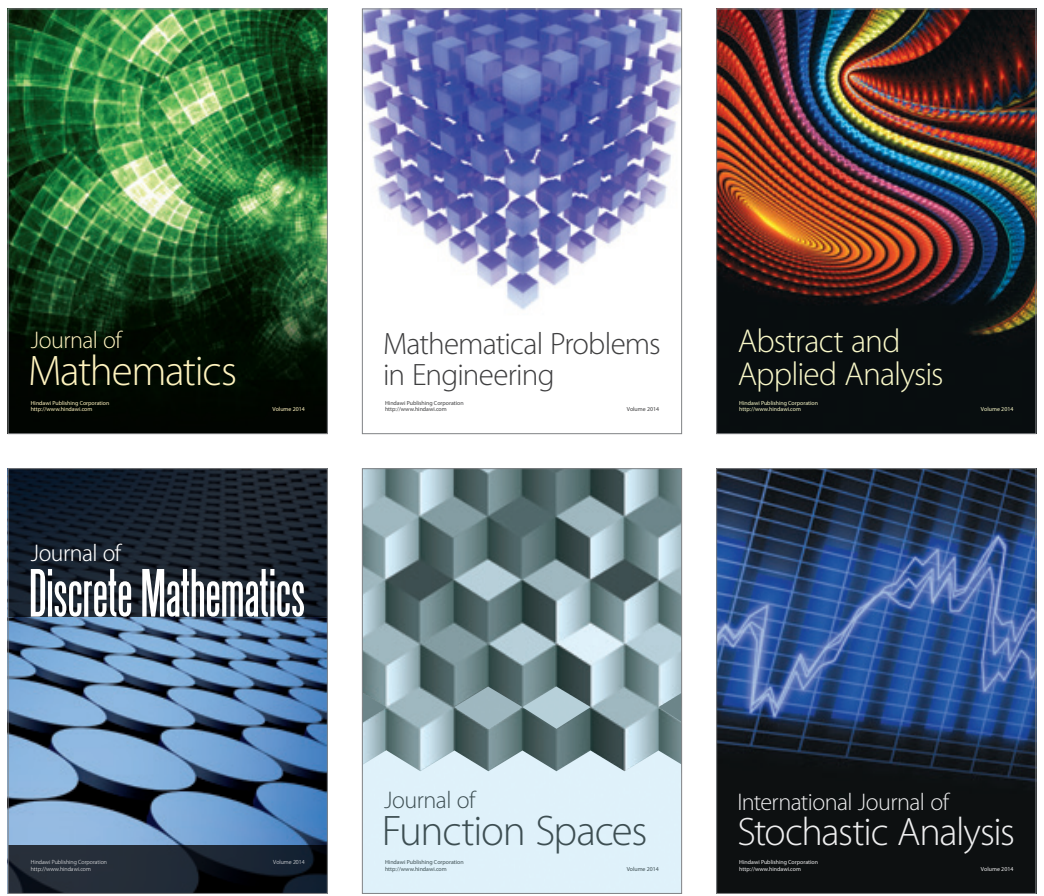

Journal of

Function Spaces

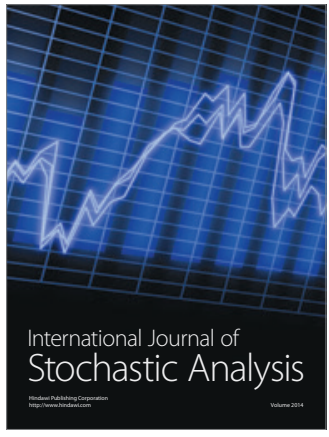

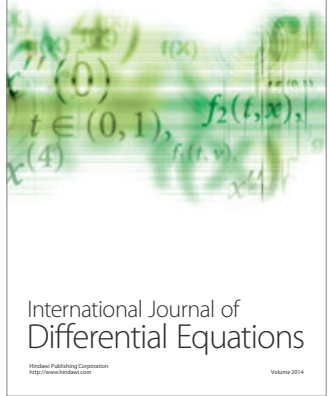
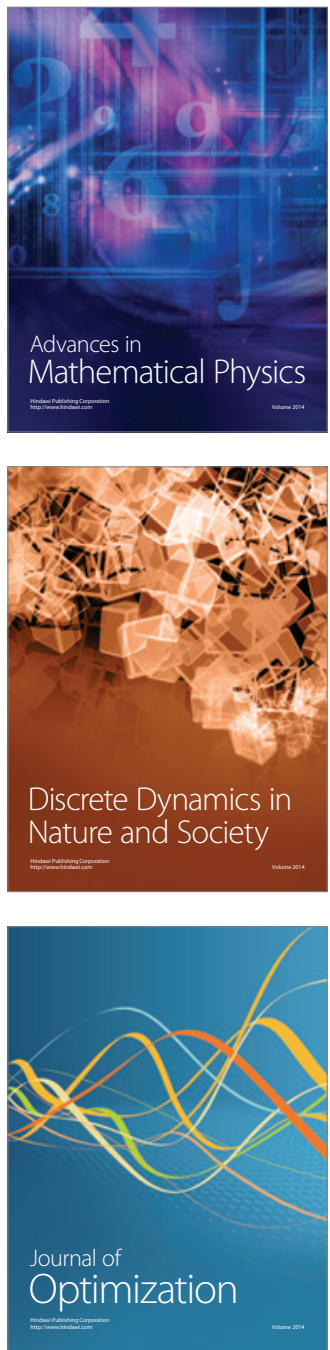